\title{
ON THE MAXIMUM \\ OF THE GEOMETRIC MOVING AVERAGE
}

\author{
A. M. HASOFER
}

(Received 17 October 1968)

\section{Introduction}

By the geometric moving average of the independent, identically distributed random variables $\left\{X_{n}\right\}$, we mean the stochastic process

$$
S_{n}=a^{n} z+a^{n-1} X_{1}+\cdots+X_{n},
$$

where $a$ is a real number such that $0 \leqq a \leqq 1$.

This process presents itself naturally in physical situations involving exponential decay. For example, it represents the response of an overdamped dynamical system to a random impulse applied at regular intervals, or the charge of a condenser which discharges into a resistance and into which is fed a random impulse at regular intervals.

Second order properties of this process are very easy to calculate and have been known for a long time. However, in many cases we are rather interested in the maximum of $S_{n}$ over an interval, i.e. in the process

$$
M_{n}=\max \left(z, S_{1}, S_{2}, \cdots, S_{n}\right) .
$$

The distribution of the tail of $M_{n}$ is required for instance for the rational design of the overdamped dynamical system. In the case of the condenser, it might be required to find the distribution of the first time that the voltage of the condenser exceeds some threshold value. This problem has been extensively studied for the case $a=0$, i.e. the case of independent random variables (see Gumbel [2]) and for the case $a=1$, i.e. the case of the random walk on the line (see Spitzer [4]).

In this paper it is first shown that the geometric moving average is characterized by being the only non-trivial Markovian moving average. Formulae for the characteristic function of $S_{n}$ and recurrence relations for its distribution are then obtained. The corresponding recurrence relations for the distribution of $M_{n}$ are then derived. They are used to obtain explicit expressions in the case where $X_{n}$ is exponentially distributed. Finally an approximate expression for the tail of the distribution of $M_{n}$ in the lastmentioned case is obtained and an upper bound for the relative error calculated. 


\section{Markovian moving averages}

The general definition of a moving average is as follows. Let $\left\{X_{n}\right\}$ be a sequence of independent, identically distributed random variables, and let $a_{0}=1,\left\{a_{n}\right\}, n>0$, be a sequence of real numbers. Then the moving average process $\left\{S_{n}\right\}$ is defined by

$$
S_{n}=a_{n} z+\sum_{k=1}^{n} a_{n-k} X_{k}, \quad(n=0,1,2, \cdots) .
$$

The process $\left\{S_{n}\right\}$ is generally non-Markovian. In fact we presently prove the following.

THEOREM. The only non-trivial Markovian moving average is the 'geometric' moving average for which $a_{n}=a^{n}$.

Proof. We can write the Markov property of the process in the form (1) $E\left[\exp \left(i p S_{n}\right) \mid S_{n-1}=s_{n-1}\right]=E\left[\exp \left(i p S_{n}\right) \mid S_{k}=s_{k}, k=1, \cdots, n-1\right]$.

Let $x_{k},(k=1, \cdots, n-1)$, be the (unique) solution of the set of equations $a_{r} z+\sum_{k=1}^{r} a_{r-k} x_{k}=s_{k}, r=1, \cdots, n-1$. Then the condition $S_{k}=s_{k}$, $(k=1, \cdots, n-1)$ is equivalent to the condition $X_{k}=x_{k},(k=1, \cdots, n-1)$. It follows that

$$
\begin{aligned}
& E\left[\exp \left(i p S_{n}\right) \mid S_{k}=s_{k}, k=1, \cdots, n-1\right] \\
& =E\left[\exp \left(i p X_{n}\right)\right] \exp \left[i p\left(a_{n} z+\sum_{k=1}^{n-1} a_{n-k} x_{k}\right)\right] .
\end{aligned}
$$

Also

$$
\begin{aligned}
& E\left[\exp \left(i p S_{n}\right) \mid S_{n-1}=s_{n-1}\right] \\
& \quad=E\left[\exp \left(i p X_{n}\right)\right] E\left\{\exp \left[i p\left(a_{n} z+\sum_{k=1}^{n-1} a_{n-k} X_{k}\right)\right] \mid S_{n-1}=s_{n-1}\right\} .
\end{aligned}
$$

Writing $X_{k}^{\prime}=X_{k}-x_{k}, S_{n}^{\prime}=\sum_{k=1}^{n} a_{n-k} X_{k}^{\prime}$, we find that equation (1) reduces to

$$
E\left\{\exp \left[i p \sum_{k=1}^{n-1} a_{n-k} X_{k}^{\prime}\right] \mid S_{n-1}^{\prime}=0\right\}=1 .
$$

In other words, the condition

must imply

$$
\sum_{k=1}^{n-1} a_{n-k-1} X_{k}^{\prime}=0
$$

$$
\sum_{k=1}^{n-1} a_{n-k} X_{k}^{\prime}=0
$$

almost surely. For $n=3$ we find that $X_{2}^{\prime}+a_{1} X_{1}^{\prime \prime}=0$ implies 


$$
a_{1} X_{2}^{\prime}+a_{2} X_{1}^{\prime}=0
$$

a.s. This can obviously happen only if $a_{2}=a_{1}^{2}$, or if the $\left\{X_{n}\right\}$ are degenerate random variables. By taking larger values of $n$ we can show by induction that $a_{n}=a^{n}$. Conversely, if $a_{n}=a^{n}$, we have $S_{n+1}=X_{n+1}+a S_{n}$, which shows that $S_{n}$ is Markovian. This completes the proof of the theorem.

In the sequel, we shall restrict ourselves to moving averages for which $a_{n}=a^{n}$. These we shall call 'geometric moving averages'.

\section{The distribution of the geometric moving average}

Let us write explicitly

$$
S_{n}(z)=a^{n} z+a^{n-1} X_{1}+a^{n-2} X_{2}+\cdots+a X_{n-1}+X_{n} .
$$

Let $\phi(t)=E\left\{\exp \left(\right.\right.$ it $\left.\left.X_{n}\right)\right\}$. Then $E\left\{\exp \left(\right.\right.$ it $\left.\left.a^{n} X_{n}\right)\right\}=\phi\left(a^{n} t\right)$, and

$$
E\left\{\exp \left[i t S_{n}(z)\right]\right\}=e^{i t a^{n} z} \prod_{k=0}^{n-1} \phi\left(a^{k} t\right)
$$

In this way we can calculate explicitly the characteristic function of $S_{n}(z)$. Also let us write $F(x)=P\left\{X_{n}<x\right\}, G_{n}(z, x)=P\left\{S_{n}(z)<x\right\}$. Then the Chapman-Kolmogorov forward and backward equations provide us with two recurrence relations, namely

and

$$
G_{n+1}(z, x)=\int_{-\infty}^{+\infty} G_{n}\left(z, \frac{x-y}{a}\right) d F(y)
$$

$$
G_{n+1}(z, x)=\int_{-\infty}^{+\infty} G_{n}(y, x) d_{y} F(y-a z)
$$

Let $\mu=E\left(X_{n}\right)$. Then

$$
\vec{S}_{n}=E\left(S_{n}\right)= \begin{cases}a^{n} z+\frac{1-a^{n}}{1-a \mu}, & \text { if } a \neq 1, \\ z+n \mu, & \text { if } a=1 .\end{cases}
$$

Let now $R_{p}$ be the central moment of order $p$ of $X_{n}$.

The central moment of order $p$ of $S_{n}$ will then be given by

$$
E\left[\left(S_{n}-\bar{S}_{n}\right)^{p}\right]= \begin{cases}\frac{1-a^{n p}}{1-a^{p}} R_{p}, & \text { if } a \neq 1, \\ n R_{p}, & \text { if } a=1 .\end{cases}
$$

If we let $n \rightarrow \infty$, we see that, if $|a|=1$, and $R_{p} \neq 0, E\left[\left(S_{n}-\bar{S}_{n}\right)^{p}\right]$ diverges. If, however, $|a|<1$ and $R_{p}$ is finite, $E\left[\left(S_{n}-\bar{S}_{n}\right)\right]^{p}$ tends to the finite limit 
$R_{p} /(1-a)$. This is true in particular for the variance of $S_{n}$, provided that the variance of $X_{n}$ is finite. It follows that in this case the distribution of $S_{n}$ tends to a limit distribution (See the theorem on infinite convolutions in Feller [1, p. 259]).

We shall now obtain an explicit formula for the distribution of $S_{n}(z)$ when $X_{n}$ has the exponential distribution with unit parameter. In that case we have $F(x)=1-e^{-x}, \phi(t)=1 /(1-i t)$.

It follows that

$$
E\left\{\exp \left[i t S_{n}(z)\right]\right\}=e^{i t a^{n} z} \prod_{k=0}^{n-1}\left(\frac{1}{1-i a^{k} t}\right) .
$$

Using the partial fraction expansion of the product, we find

$$
E\left\{\exp \left[i t S_{n}(z)\right]\right\}=\sum_{r=0}^{n-1}\left[\prod_{k=0}^{n-1} \frac{1}{1-a^{k-r}}\right] \frac{e^{i t a^{n} z}}{1-i a^{r} t},
$$

where $\Pi^{\prime}$ denotes taking a product over all values of $k$ except $k=r$. Inverting this expression, we find that

$$
\left.G_{n}(z, x)=1-\sum_{r=0}^{n-1}\left[\prod_{k=0}^{n-1} \frac{1}{1-a^{k-r}}\right] \exp \left(-\frac{x}{a^{r}}+a^{n-r} z\right)\right) .
$$

For example, if $n=3$, we find that

$$
G_{3}(z, x)=1-\frac{e^{a^{3} z}}{(1-a)\left(1-a^{2}\right)} e^{-x}+\frac{a e^{a^{2} z}}{(1-a)^{2}} e^{-x / a}-\frac{a^{3} e^{a z}}{(1-a)\left(1-a^{2}\right)} e^{-x / a^{2}} .
$$

\section{The distribution of the maximum of $S_{n}$ for $0<a<1$}

We now write

$$
M_{n}(z)=\max \left[S_{0}(z), S_{1}(z), \cdots, S_{n}(z)\right], \text { where } S_{0}(z)=z,
$$

and we restrict ourselves to values of $a$ satisfying $0<a<1$.

We cannot study the distribution of $M_{n}(z)$ directly, because it is not a Markovian process. We therefore consider instead the first passage time of $S_{n}(z)$ at $h$. Let $N_{h}(z)$ be this first passage time. Then

$$
\left\{N_{h}(z)=n\right\} \equiv\left\{S_{r}(z)<h, i=0,1, \cdots, n-1 ; S_{n}(z) \geqq h\right\} .
$$

This can be rewritten

$$
\left\{N_{h}(z)=n\right\} \equiv\left\{M_{n-1}(z)<h ; M_{n}(z) \geqq h\right\} .
$$

It follows that

$$
\left\{N_{h}(z)>n\right\} \equiv\left\{M_{n}(z)<h\right\},\left\{N_{h}(z) \geqq n\right\} \equiv\left\{M_{n-1}(z)<h\right\} .
$$


(Note that $N_{h}(z)$ is a defective random variable and may equal $+\infty$ with finite probability).

Let us now write

$$
\begin{aligned}
Q_{n}(z, x) & =P\left\{S_{n}(z)<x ; N_{h}(z) \geqq n\right\} \\
& =P\left\{S_{n}(z)<x ; M_{n-1}(z)<h\right\} .
\end{aligned}
$$

Then we can see that

$$
\begin{aligned}
Q_{n}(z, h) & =P\left\{S_{n}<h ; M_{n-1}(z)<h\right\} \\
& =P\left\{M_{n}(z)<h\right\} .
\end{aligned}
$$

The best tool for calculating $Q_{n}(z, h)$ is the backward Kolmogorov equation

$$
Q_{n+1}(z, h)=U(h-z) \int_{-\infty}^{h-} Q_{n}(y, h) d_{y} F(y-a z), \quad n=0,1, \cdots,
$$

where $U(x)=1$ for $x>0$ and $U(x)=0$ for $x \leqq 0$. (See Kemperman $\left[3\right.$, p. 55]). Also, we obviously have $Q_{0}(z, x)=U(x-z)$. Thus $Q_{n}(z, h)$ can be calculated by iteration. However, unlike the case $a=1$ (i.e., the case of the random walk), it does not seem possible to obtain a closed formula for the generating function of the $Q_{n}$.

It is important to note that if the $X_{n}$ are non-negative, formula (6) can be written in a slightly more manageable form which does not involve an infinite interval of integration. In that case we can write the distribution function of $X_{n}$ in the form $F(x) U(x)$. Let us also assume that the distribution of $X_{n}$ has no concentration of probability at the origin and has a probability density $f(x)$.

Then equation $(6)$ reduces to

$$
Q_{n+1}(z, h)=U(h-z) \int_{a z}^{n} Q_{n}(y, h) f(y-a z) d y .
$$

\section{The case of exponentially distributed $X_{n}$}

We shall now obtain explicit expressions for the distribution of $M_{n}(z)$ in the case when $X_{n}$ is exponentially distributed with unit parameter, that is, when $F(x)=1-e^{-x}, f(x)=e^{-x}$. There is no loss of generality in assuming unit parameter for the distribution, as only a change of scale is involved.

We easily find

$$
\begin{aligned}
& Q_{1}(z, h)=\left(1-e^{-h+a z}\right) U(h-z), \\
& Q_{2}(z, h)=\left[1-e^{-h}\left\{\left(1-\frac{e^{-(1-a) h}}{1-a}\right) e^{a z}+\frac{e^{a^{2} z}}{1-a}\right\}\right] U(h-z) .
\end{aligned}
$$


This suggests that $Q_{n}(z, h)$ is of the form

$$
Q_{n}(z, h)=\left(1-e^{-n} \sum_{k=1}^{n} C_{k}^{(n)} e^{a^{k} z}\right) U(h-z) .
$$

It is easily checked that this form of $Q_{n}(z, h)$ satisfies the recurrence relation (6), provided the coefficients $C_{k}^{(n)}$ satisfy the recurrence relations

$$
\begin{array}{ll}
C_{1}^{(n+1)}=\left(1-\sum_{k=1}^{n} \frac{C_{k}^{(n)}}{1-a^{k}} e^{-\left(1-a^{k}\right) h}\right), & \\
C_{k}^{(n+1)}=\frac{C_{k-1}^{(n)}}{1-a^{k-1}}, & k>1 .
\end{array}
$$

If we write $b_{k}=1 /\left(1-a^{k}\right)$, we can rewrite relations (9) in the form

$$
\begin{aligned}
C_{1}^{(n+1)} & =1-\sum_{k=1}^{n} C_{k}^{(n)} b_{k} e^{-n / b_{k},} & \\
C_{k}^{(n+1)} & =b_{k-1} C_{k-1}^{(n)}, & k>1 .
\end{aligned}
$$

Let us write $C^{(n)}=\left(1, C_{1}^{(n)}, C_{2}^{(a)}, \cdots, C_{n}^{(n)}, 0, \cdots\right)$, a row vector of infinite length. Then

$$
C^{(0)}=(1,0,0, \cdots) \text {. }
$$

The recurrence relations (9a) can be written in matrix form

$$
C^{(n+1)}=C^{(n)} P,
$$

where $\boldsymbol{P}$ is the infinite matrix

$$
A=\left(\begin{array}{ccccc}
1 & 1 & 0 & 0 & \cdots \\
0 & -b_{1} e^{-n / b_{1}} & b_{1} & 0 & \cdots \\
0 & -b_{2} e^{-n / b_{2}} & 0 & b_{2} & \cdots \\
\ldots & \ldots & \ldots & \ldots & \ldots
\end{array}\right)
$$

In equation (10) as well as in all matrix equations in the sequel, we shall take as the norm of the matrix $A=\left(a_{i j}\right)$ the value $\|A\|=\max _{i}\left(\Sigma_{j} a_{i j}\right)$. It is obvious that we have $\|A\|<2 /(1-a)$.

From equation (10), we immediately deduce that

Finally, if we write

$$
C^{(n)}=C^{(0)} A^{n} \text {. }
$$

$$
z=\left(1,-e^{-h+a z},-e^{-\lambda+a^{2} z}, \cdots\right)^{\prime},
$$

where the dash denotes transposition, we see that for $0 \leqq z<h$,

$$
Q_{n}(z, h)=C^{(n)} z=C^{(0)} A^{n} z
$$


From expression (11) we can calculate immediately the generating function of the $Q_{n}$. In fact we have

$$
\begin{aligned}
Q(z, h) & =\sum_{n=0}^{\infty} t^{n} Q_{n}(z, h)=\boldsymbol{C}^{(0)}\left[\sum_{n=0}^{\infty} t^{n} \boldsymbol{A}^{n}\right] z, \\
& =\boldsymbol{C}^{(0)}(\boldsymbol{I}-\boldsymbol{A} t)^{-1} \boldsymbol{z},
\end{aligned}
$$

where $I$ denotes an infinite unit matrix. The inverse matrix $(\boldsymbol{I}-\boldsymbol{A} t)^{-\mathbf{1}}$ will certainly exist at least for $\|t\|<\|\boldsymbol{A}\|^{-1}$, that is, at least for $|t|<(1-a) / \mathbf{2}$.

We can calculate the elements $\left(x_{i j}\right)$ of the matrix $X=(I-A t)^{-1}$ by just writing down the scalar elements of the defining equation

$$
\boldsymbol{X}(\boldsymbol{I}-\boldsymbol{A} t)=\boldsymbol{I} .
$$

However, in view of the fact that we are only interested in the product $C^{(0)}(I-A t)^{-1}$, which is actually only the first row of $(I-A t)^{-1}$, it is necessary to calculate only $x_{1 n}, n=1,2, \cdots$. We find

$$
\begin{aligned}
& x_{11}=\frac{1}{1-t}, \\
& x_{1 n}=\frac{h_{n-1} t^{n-1}}{(1-t) \sum_{n=0}^{\infty} k_{n} t^{n}},
\end{aligned}
$$

where

$$
\begin{aligned}
& h_{n}=\frac{1}{(1-a)\left(1-a^{2}\right) \cdots\left(1-a^{n-1}\right)}, \\
& k_{n}=\frac{e^{-\left(1-a^{n}\right) h}}{(1-a)\left(1-a^{2}\right) \cdots\left(1-a^{n}\right)}, \quad(n=1,2, \cdots)
\end{aligned}
$$

and $k_{0}=1$.

It immediately follows that

$$
\begin{aligned}
Q(z, h) & =\boldsymbol{C}^{(0)}(\boldsymbol{I}-\boldsymbol{A} t)^{-1} z, \\
& =\frac{1}{1-t}-\frac{e^{-h}}{1-t} \frac{\sum_{n=1}^{\infty} e^{a^{n} z} h_{n} t^{n}}{\sum_{n=0}^{\infty} k_{n} t^{n}} .
\end{aligned}
$$

Let us denote by $D(t)$ the series $\sum_{n=0}^{\infty} k_{n} t^{n}$. Then

$$
|1-D(t)|=\left|\sum_{n=1}^{\infty} k_{n} t^{n}\right| \leqq e^{-(1-a) h} \sum_{n=1}^{\infty}\left|\frac{t}{1-a}\right|^{n}=\frac{|t|}{b-|t|} e^{-b n},
$$

where $b=1-a$.

It follows that $D(t)$ has no zeros at least for $|t|<b /\left(1-e^{-b h}\right)$.

It is also easily ascertainable that the series $N(t)=\sum_{n=0}^{\infty} e^{a^{n} z} h_{n} t^{n}$ has a radius of convergence of unity. Thus equation (12) does actually define an analytic function of $t$ in the neighbourhood of the origin. 


\section{An asymptotic formula for large $h$}

It is clear that for very large $h$ the series $D(t)$ will be very nearly equal to one. We therefore conjecture that the coefficients of the generating function $Q(z, h)$ will be approximated for large $h$ by those of

$$
Q^{*}(z, h)=\frac{1}{1-t}-e^{-h} \frac{N(t)}{1-t} .
$$

It is easy to see that the required coefficients $Q_{n}^{*}(z, h)$ are given by

$$
\begin{aligned}
& Q_{0}^{*}(z, h)=1, \\
& Q_{n}^{*}(z, h)=1-e^{-h}\left[e^{a z}+\frac{e^{a^{2} z}}{1-a}+\cdots+\frac{e^{a^{n} z}}{(1-a) \cdots\left(1-a^{n-1}\right)}\right], \quad n \geqq 1 .
\end{aligned}
$$

To evaluate the goodness of the approximation, we note that

$$
\sum_{n=0}^{\infty}\left(Q_{n}-Q_{n}^{*}\right) t^{n}=\frac{e^{-n} N(t)}{(1-t)}\left[\frac{1-D(t)}{D(t)}\right] .
$$

It follows that for $R<b /\left(1-e^{-b h}\right)$,

$$
Q_{n}-Q_{n}^{*}=\frac{e^{-h}}{2 \pi i} \int_{|t|=R} \frac{N(t)}{t^{n+1}(1-t)} \frac{1-D(t)}{D(t)} d t .
$$

We notice that

$$
\begin{aligned}
& |N(t)| \leqq(1-a) e^{a z} \sum_{n=1}^{\infty}\left|\frac{t}{1-a}\right|^{n}=\frac{b|t|}{b-|t|} e^{a z}, \\
& |D(t)| \geqq 1-|1-D(t)| \geqq 1-\frac{|t|}{b-|t|} e^{-b h}=\frac{b-|t|\left(1-e^{-b h}\right)}{b-|t|} .
\end{aligned}
$$

We can therefore conclude from (14) that

$$
\begin{aligned}
\left|Q_{n}-Q_{n}^{*}\right| & \leqq \frac{b e^{-(1+b) h+a z}}{R^{n-1}(1-R)(b-R)\left[b-R\left(1-e^{-b h}\right)\right]}, \\
& <\frac{b e^{-(1+b) h+a z}}{R^{n-1}(b-R)^{3}} .
\end{aligned}
$$

The maximum value of the last denominator is $27 n^{3} b^{n+3}[(n+3) / n]^{-(n+3)}$, which for large $n$ is approximately equal to $27(n / e)^{3} b^{n+3}$.

Thus we eventually obtain the inequality

$$
\left|Q_{n}-Q_{n}^{*}\right|<\frac{e^{-(1+b) h+a z+3}}{27 n^{3} b^{n+3}}
$$

This shows that as $h \rightarrow \infty,\left|Q_{n}-Q_{n}^{*}\right| \rightarrow 0$. We are, however, more interested 
in the relative error, as both $Q_{n}$ and $Q_{n}^{*}$ tend to one as $h$ tends to infinity. It is obvious from equation (13) that

Thus we have

$$
\left|1-Q_{n}^{*}\right|>e^{-n+a z} \text {. }
$$

$$
\left|\frac{\left(1-Q_{n}\right)-\left(1-Q_{n}^{*}\right)}{1-Q_{n}^{*}}\right| \leqq \frac{e^{-b n+3}}{27 n^{3} b^{n+3}}
$$

Thus the relative error in $1-Q_{n}$ also tends to zero for large $h$.

\section{References}

[1] W. Feller, $A n$ introduction to probability theory and its applications (Vol. II., Wiley \& Sons, New York, 1966).

[2] E. J. Gumbel, Statistics of extremes (Columbia University Press, New York, 1958).

[3] J. H. B. Kemperman, The passage problem for a stationary Markov chain (University of Chicago Press, 1961).

[4] F. Spitzer, Principles of Random Walk (Van Nostrand, Princeton, 1964).

Australian National University

Canberra, Australia 